\title{
Examination of Edge Effects with Different Storage Conditions of Preplated Dimethyl Sulfoxide Nanospots in ChemLib 1,536- and 3,456-Well Assay-Ready Plates
}

\author{
Benjamin A. Turner, ${ }^{1, *}$ Brad P. Evans, ${ }^{2, \dagger}$ Tania T. Pearson, ${ }^{1,+}$ \\ Timothy K. Braden, ${ }^{1, 末}$ and Scott C. Wise ${ }^{1, \S}$
}

\begin{abstract}
For ultra-high-throughput screening, 10-30 nl of compound dissolved in 75\% dimethyl sulfoxide (DMSO) $/ 25 \%$ water ( $\mathrm{vol} / \mathrm{vol}$ ) is spotted into 1,536 - and 3,456-well ChemLib ${ }^{\mathrm{TM}}$ plates (Aurora Biotechnologies, Carlsbad, CA) and stored appropriately for a short time before screening. Although this practice eliminates the compound plating bottleneck, plated volumes of DMSO slowly evaporate from assay wells if plates are not properly stored in the interim. Since many assays are sensitive to DMSO concentrations, even slight evaporation may cause intra-plate variation and thus decrease assay quality. Using a cytochrome P450 3A4 Vivid ${ }^{\circledR}$ Blue assay (Invitrogen, Carlsbad), we investigated the rate, pattern, and quantity of evaporation over a 1-year time frame to identify best practices for long-term (i.e., 6 months or greater) storage of assay-ready compound plates. Our findings regarding evaporation at plate edges indicate that nanospots preplated in ChemLib 1,536- or 3,456 -well plates are best stored at $-80^{\circ} \mathrm{C}$, in a bag, with or without the outer evaporation wells filled or at $-20^{\circ} \mathrm{C}$, in a bag, with evaporation wells filled.
\end{abstract}

\section{Introduction}

CREENING COMPOUNDS are routinely dissolved in dimethyl sulfoxide (DMSO) for both biochemical and cell-based assays. Companies have therefore collaborated on and/or published a number of studies designed to characterize compound stability under various conditions, ${ }^{1-4}$ quantify hydration of solvent in chemical libraries, ${ }^{5}$ and evaluate the extent of solubilization. ${ }^{6}$ Few studies have been published on evaporation of preplated solvent nanospots in high-density assay-ready microplates, namely, 1,536- and 3,456-well plates, despite the complications that result from deviations in well-to-well solvent volumes. Most biological assays tolerate the presence of the solvent, but with en- zymes or cells sensitive to its concentration at or near typical screening concentrations, edge effects become increasingly predominant ${ }^{7}$ with extended storage durations. Accordingly, some form of rigorous statistical manipulation is necessary to smooth out such effects, which extends data package delivery timelines.

In our laboratory, preplating and appropriately storing compounds have helped achieve significant resource savings. First, assay-ready plates allow for greater daily throughput and thus more efficient screening of the chemical library. Same-day plating practices, in contrast, either extend working hours or limit the assay throughput - particularly because plating times significantly exceed reagent addition times with our hardware. Second, standardizing

Departments of ${ }^{1}$ Assay Technologies and ${ }^{2}$ Statistics, Pfizer Global Research and Development, Michigan Laboratories, Ann Arbor, Michigan.

*Present address: Gilead Colorado, Westminster, Colorado.

†Present address: Pfizer Global Research and Development, Groton, Connecticut.

¥Present address: Pfizer Global Research and Development, St. Louis, Missouri.

§Present address: Deciphera Pharmaceuticals, Lawrence, Kansas.

ABBREVIATIONS: CYP3A4, cytochrome P450 3A4; DMSO, dimethyl sulfoxide; FRD, flying reagent dispenser; NADP ${ }^{+}$, nicotinamide adenine dinucleotide phosphate ion; PSDR, piezo sample distribution robot. 
the throughput of a screen facilitates planning - suitable numbers of cells are cultured, costly reagent dead-volumes are minimized, and productivity is optimized. And, with our hardware, plating multiple copies of plates enhances productivity even further because of liquid handling dynamics. To illustrate, the Aurora Discovery Inc. (San Diego, CA) piezo sample distribution robot $(\mathrm{PSDR})^{8} \mathrm{de}-$ votes little time to aspirating and dispensing compounds and plate handling, but much time to tip washing between compound delivery cycles. Accordingly, plating four or more copies of the compound library requires little extra time than that devoted to plating a single copy, ${ }^{9}$ and plates can be stored appropriately for an extended period of time. Assays ready for screening after assay development and adaptation can then be screened without the "bottleneck" of compound plating.

In the presence of high atmospheric humidity, DMSO rapidly equilibrates with water to about $75 \%$ by volume. ${ }^{10}$ For that reason, $25 \%(\mathrm{vol} / \mathrm{vol})$ water was added to the screening library source plates in our laboratory followed by room temperature storage in a bag (with a Petri dish, as described below) to eliminate water absorption and keep both the solvent viscosity and well volumes constant (P.J. Coassin, Aurora Discovery Inc., personal communication). Recent publications have detailed the effects water has upon higher (10 $\mathrm{m} M$ and $30 \mathrm{mM})$ concentrations of some compound classes, ${ }^{1-4}$ but deleterious effects decrease at lower compound concentrations. ${ }^{6}$ Further, we empirically observed that the presence of $25 \%$ water eliminated freezethaw cycles when assay plates were stored briefly at $-20^{\circ} \mathrm{C}$ prior to use, since the $75 \% \mathrm{DMSO} / 25 \%$ water mix remained in the liquid phase at that temperature. With small batches of compounds, preparation of $100 \%$ DMSO nanospots is of course possible, accordingly eliminating concerns over water's deleterious effects at any compound concentration and the need for source plate filtration prior to storage.

Here we describe the use of a cytochrome P450 3A4 (CYP3A4) fluorogenic assay ${ }^{11}$ (Invitrogen, Carlsbad, CA) to quantify the plate edge evaporation of $75 \%$ $\mathrm{DMSO} / 25 \%$ water nanospots stored under various conditions. In the absence of DMSO, CYP3A4 readily cleaves the nonfluorescent dye to yield a fluorescent product, whereas DMSO inhibits the reaction in a concentration-dependent manner. Accordingly, final DMSO concentration changes of $0.1 \%$ due to solvent evaporation can be detected. ChemLib ${ }^{\mathrm{TM}}$ plates (Aurora Biotechnologies, Carlsbad) were selected for the study because of the design of both plate, with fillable additional perimeter wells, and lid for evaporation control (Fig. 1).

\section{Materials and Methods}

Assay plates are from Aurora Biotechnologies and reagents from Invitrogen, except where noted. Assay

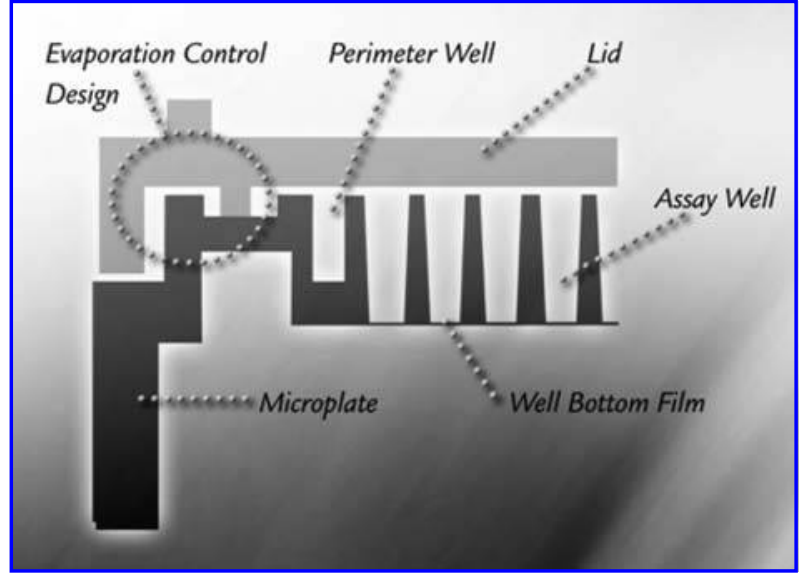

FIG. 1. ChemLib plate and lid design for evaporation control. ${ }^{12}$

plates were prepared on the PSDR such that each well of ChemLib 1,536- or 3,456-well black-wall/clear-bottom plates contained $20 \mathrm{nl}$ or $10 \mathrm{nl}$, respectively, of $75 \%$ $\mathrm{DMSO} / 25 \%$ water for evaluation of the following storage conditions:

1. $-20^{\circ} \mathrm{C}$, without bag

2. $-20^{\circ} \mathrm{C}$, without bag, with filled evaporation wells

3. $-20^{\circ} \mathrm{C}$, with bag

4. $-20^{\circ} \mathrm{C}$, with bag, with filled evaporation wells

5. $-20^{\circ} \mathrm{C}$, with bag, with PlateLoc ${ }^{\circledR} \mathrm{Al} /$ polymer seal

(Velocity11, Menlo Park, CA)

6. $-80^{\circ} \mathrm{C}$, with bag

7. $-80^{\circ} \mathrm{C}$, with bag, with filled evaporation wells

Plates were passed over a static neutralizing curtain transvector (SIMCO, Hatfield, PA) to remove dust and static prior to use; perimeter evaporation wells (Fig. 1) were filled with either $5.0 \mu \mathrm{l}(1,536$-well) or $1.5 \mu \mathrm{l}$ $(3,456$-well) of the same $75 \% \mathrm{DMSO} / 25 \%$ water mix. Saranex ${ }^{\circledR}$ vapor-barrier bags (Thermo Fisher Scientific, Waltham, MA) contained Petri dishes (Kimble Glass Inc., Vineland, NJ) filled with $3 \mathrm{ml}$ of the $75 \% \mathrm{DMSO} / 25 \%$ water mix. Sealed plates were prepared using machined metal plate shims to ensure uniform seals and sealed at a temperature of $160^{\circ} \mathrm{C}$ for $2 \mathrm{~s}$. Control plates were prepared using the PSDR such that each well contained 0 , $4,16,24$, or $36 \mathrm{nl}$ of $75 \% \mathrm{DMSO} / 25 \%$ water for 1,536 well studies and $0,2,3,5,9$, or $18 \mathrm{nl}$ of the mix in 3,456well studies. Plates were stored either at room temperature in a bag for use within $2 \mathrm{~h}$ or at $-20^{\circ} \mathrm{C}$ in a bag with a dish for use the next day. On occasions when the PSDR was not available for control plate preparation, the picoliter rapid transfer robot (PicoRapTR ${ }^{\mathrm{TM}}$, Beckman Coulter, Fullerton, CA) was substituted, which uses the same piezo tip dispensing technology as the PSDR. 
Assay reagents were dispensed first into the control plate and then into test plates, in the order of storage conditions listed above, using the flying reagent dispenser $(\mathrm{FRD})^{8}$ (now the biological rapid transfer robot [BioRapTR $^{\mathrm{TM}}$ ], Beckman Coulter) fitted with either a 1,536well or 3,456-well head. Assay volumes were either 3.0 $\mu \mathrm{l}$ (1,536-well) or $1.5 \mu \mathrm{l}$ (3,456-well). Prior to preparing assay reagents, plates were removed from storage conditions and set out to equilibrate to room temperature for 45-60 min; plates not stored in bags were equilibrated in bags to prevent solvent evaporation during set-up. A chiller (Brinkmann, Mississauga, ON, Canada) adapted with a custom water jacket and circulation tubing was used to maintain the enzyme mix at $\sim 4^{\circ} \mathrm{C}$. Dispense tables were set-up to include $100 \mu \mathrm{l}$ of reagent priming prior to dispensing. Reagents were loaded onto the hardware such that reactions were initiated with the addition of enzyme. Reactions consisting of DMSO (ideally $0.5 \%), 5 \mathrm{n} M$ CYP3A4 enzyme, $20 \mu M$ CYP3A4 Blue substrate (BOMCC), $100 \mu M$ nicotinamide adenine dinucleotide phosphate ion $\left(\mathrm{NADP}^{+}\right)$, and $1 \times$ regeneration system in $1 \times$ reaction buffer were incubated for $20 \mathrm{~min}$ at room temperature. Plates were read on the topology compensating plate reader ${ }^{8}$ (Aurora Discovery) using 400 $\mathrm{nm}$ excitation and $460 \mathrm{~nm}$ emission filters (Chroma Technologies, Rockingham, VT) immediately after addition of assay stop mix.

Ketoconazole (Sigma-Aldrich, St. Louis, MO) was prepared as a $1 \mathrm{~m} M$ stock solution in $100 \%$ DMSO, protected from light, and stored either at room temperature for usage within $1-2$ months or at $-20^{\circ} \mathrm{C}$ for later use. Frozen stocks were removed from the freezer at least 24 $\mathrm{h}$ prior to use to prevent precipitation upon addition to phosphate-buffered saline. The assay stop mix, $180 \mu M$ $(5 \times)$ ketoconazole in phosphate-buffered saline, was prepared and stored at room temperature protected from light until use.

Vials of CYP3A4 substrate, stored desiccated at $4^{\circ} \mathrm{C}$, were removed from the refrigerator and allowed to reach ambient temperature before uncapping. Anhydrous acetonitrile (Mallinckrodt, Hazelwood, MO) was added to each tube, the tubes were capped, and the solution was vortex-mixed to prepare a $2 \mathrm{~m} M$ stock. Stock solutions were used at room temperature but stored desiccated at $4^{\circ} \mathrm{C}$; previously reconstituted substrate was warmed to room temperature before uncapping for use. To prepare the substrate mix, $2 \times$ reaction buffer and distilled water were combined in a 50-ml foil-covered conical vial (VWR, Bridgeport, NJ), and substrate was added. Thawed $\mathrm{NADP}^{+}$(on ice) was added to the mixture prior to lidding and vortex-mixing. The mix $(4 / 3 \times$ substrate and $\mathrm{NADP}^{+}$in $1 \times$ buffer) was kept at room temperature protected from light until use.

To prepare both the enzyme mix and control (no enzyme) mix, $2 \times$ reaction buffer and distilled water were combined in a 50-ml conical vial on ice. Regeneration system reagent was allowed to thaw on ice, vortex-mixed, then added directly to the diluted reaction buffer, and mixed by further vortex-mixing. Baculosomes ${ }^{\circledR}$ (Invitrogen), thawed on ice, were added gently, and the resultant enzyme mix $(4 \times$ enzyme and regeneration system in $1 \times$ buffer) was mixed by inversion and stored on ice until use. Nalgene FRD bottles (Thermo Fisher Scientific) were chilled thoroughly prior to use as well.

Data were analyzed using Microsoft (Redmond, WA) Excel $^{\circledR}$. Initially, control plate data were graphed as percentage activity versus $\log _{10}$ of the DMSO concentration. Subsequently, percentage activity for various storage conditions was extrapolated from the DMSO sensitivity curve (Fig. 2). SAS software (SAS Institute Inc., Cary, NC) was used to compile data prior to Spotfire ${ }^{\circledR}$ (Spotfire Inc., Somerville, MA) visualization of study data.

\section{Results and Discussion}

With this assay system, DMSO inhibits CYP3A4 cleavage of the nonfluorescent blue dye to yield a fluorescent product in a concentration-dependent manner. Accordingly, final DMSO concentration changes of $0.1 \%$ due to solvent evaporation can be detected (Fig. 2). Because of this assay sensitivity, evaporative patterns were evident over time for many of the tested storage conditions, but that same sensitivity resulted in marked data fluctuations. Initially, we observed an apparent gain in DMSO across plates within a single run due to loss of enzyme activity over time (data not shown). Such data

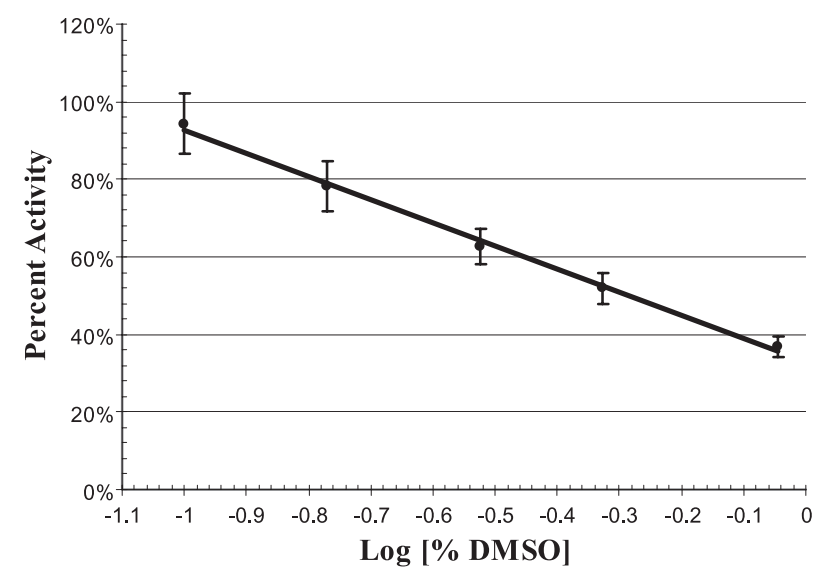

FIG. 2. Representative DMSO sensitivity curve for a 3,456well study time point. Data from control plates were used to construct a standard curve of percentage activity versus the $\log _{10}$ of DMSO concentration at each time point of the study. $y=$ $-0.6005 x+0.3277, R^{2}=0.9963$. DMSO remaining in assay plate wells for each storage condition was subsequently calculated by extrapolation. Comparable graphs were constructed for the 1,536-well study (data not shown). 
trends, which confounded DMSO amounts with enzyme activity, varied from month-to-month and study-to-study, thereby precluding the possibility of smoothing data to quantify evaporation. Much of the effect likely resulted from variance in baculosome preparation, although the mix was concentrated and chilled per peer recommendation in an effort to stabilize the enzyme.

Reagent sensitivity is further illustrated by trailing of data toward high relative activity in the first dozen or so assay plate wells (red circle in Fig. 3) resulting from contact with instrument solenoid valves between FRD dispenses. Further, despite rigorous PSDR quality control practices, tip-to-tip variance did add a level of complexity to these data, as underdispensing and/or nonfiring piezo tips were evident on some plates of some runs (green circle in Fig. 3). Upon completion of the study, however, SpotFire trellising by assay order helped differentiate between evaporation and enzyme activity effects for each condition because neighboring plates had fewer intra-day temporal effects than distant pairs. Although not perfect, predictable patterns present in the data did sufficiently allow for qualitative determinations.

For integrity of compound solvation, some solvent (DMSO/water in this case) must be present in the well. In practice, effects upon data due to some extent of evaporation can be statistically "smoothed" out when using DMSO-sensitive cells or reagents. We defined a plate's expiration date, therefore, as the time at which no DMSO remained in some significant subset of plate wells, but, ideally, at least half of the prespotted DMSO would remain for use in an assay. Given this consideration and for graphical clarity (see Figs. 3-6), only the two outermost "rings" - that is, the two perimeter rows and columns of assay plate wells - were graphed, and central plate wells omitted from consideration. Furthermore, early studies in our laboratory (data not shown) verified that evaporation proceeded from outermost to innermost wells in these high-well-density microplates. For 1,536well plates, the first ring contains 156 wells and the second 148 for a total of 304 wells. Thus, in a compound screening context, evaporation from the outermost perimeter wells would result in $10.2 \%$ of plated compounds without any solvent and $19.8 \%$ if evaporation continued to the second ring of edge wells. Evaporation did extend beyond the second ring of wells under several storage conditions, but plates with the outermost ring of wells fully evaporated would already be at or beyond the defined expiration date. Similarly, for 3,456-well plates, the first ring of wells contains 236 wells and the second 228 for a total of 464 wells, resulting in $6.8 \%$ and $13.4 \%$, respectively, of plated screening compounds out of solution. Such a large percentage of evaporated wells would be unacceptable for any high-throughput screen.

Figure 3 shows 1,536-well data from storage at $-20^{\circ} \mathrm{C}$ without a bag. Without filled evaporation wells, a "frowning" pattern (blue circle in Fig. 3) started to appear in the data at 2 months of storage as a result of evaporation at plate edges and worsened with longer storage times. In contrast, under the analogous storage condition with filled evaporation wells, data from months 2 and 3 showed little, if any, change from day 1 as indicated by a somewhat constant relative percentage of DMSO across colored piles. Effects of edge evaporation or storage time did not first appear until month 5, and about half of the original DMSO appeared present in perimeter wells at month 7 . Overall, both $-20^{\circ} \mathrm{C}$ storage conditions without a bag showed evaporation over time, but filled evaporation wells decreased both the rate of evaporation and the presence of edge effects. Interestingly, storing plates with a bag but without filled evaporation wells was worse than filling the evaporation wells but not using the bag (Fig. 4). Plates stored at $-20^{\circ} \mathrm{C}$ with a bag appeared usable for 4 months, with minor edge evaporation evident at months 3 and 4. At month 5, significant edge effects were observed, and roughly half the spot volume remained. With filled evaporation wells, the effective use of bagged plates was extended to 7 months, as in the comparable condition without the bag. Under that condition, evaporation patterns did not appear through month 6 , but at month 7, about half the spot volume remained in outer assay wells.

The 1,536-well $-20^{\circ} \mathrm{C}$ heat-sealed condition was highly variable, with six good plates and six bad plates in no particular order (data not shown). Although sealed plates were inspected for seal integrity after preparation and prior to storage, the data suggested that sealing parameters may not have been optimal for this extended study. Recentrifuging plates prior to assays might have improved the results as well but was not done because of practical considerations. In contrast to all $-20^{\circ} \mathrm{C}$ conditions considered, both $-80^{\circ} \mathrm{C}$ conditions exhibited no discernable evaporation from edge wells over the entirety of the 12-month study (data not shown), likely because of the $75 \% \mathrm{DMSO} / 25 \%$ water mix freezing at that temperature. Accordingly, such a practice would be ideal for shipping, provided one freeze-thaw cycle is permissible. Further, storage of samples in $100 \%$ DMSO may yield comparable results at a temperature of $-20^{\circ} \mathrm{C}$ after plate preparation with low atmospheric relative humidity, due to the decreased rate of equilibration with atmospheric water prior to storage and freezing (W.S. Fillers, TekCel Inc., personal communication).

Figure 5 shows 3,456 -well data from storage at $-20^{\circ} \mathrm{C}$ without a bag. Without filled evaporation wells, frowning first appeared in the outermost wells at month 4; wells still appeared usable at month 5 but significantly degraded and became unusable prior to month 6. Comparable storage with filled evaporation wells extended the acceptable storage time to a full year, the duration of the study - even without a bag. With a bag (Fig. 6) and empty evaporation wells, the characteristic edge pattern appeared first at 4 months but did not appear significantly 


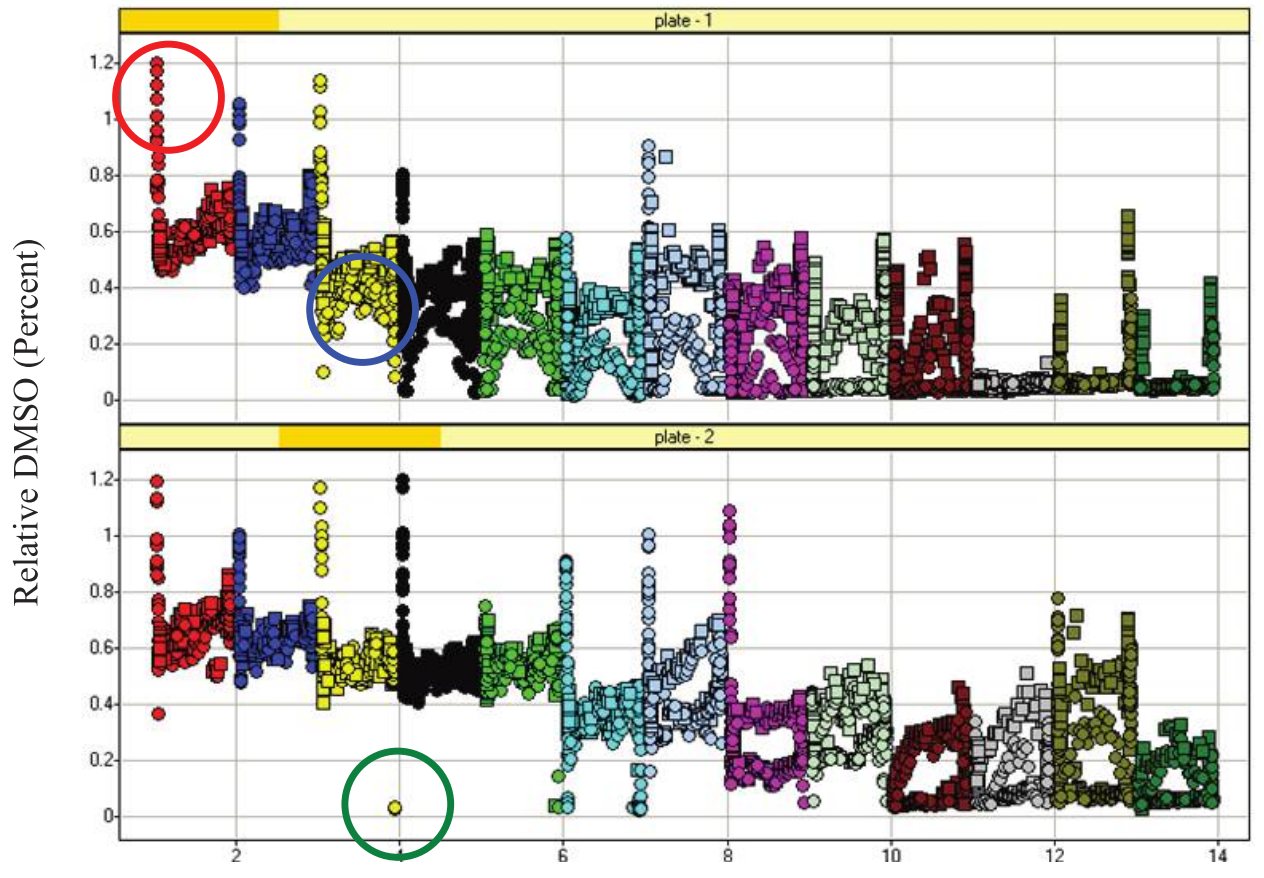

Time Point (Offset by Well Order)

FIG. 3. 1,536-well data from storage at $-20^{\circ} \mathrm{C}$ without a bag. Data from the two outermost wells about the plate are paneled by storage condition and colored by time point; data from center wells are omitted for clarity. Plate-type 1 data (top) correspond to empty evaporation wells, and plate-type 2 data (bottom) correspond to filled evaporation wells. Colored "piles" of data correspond to 13 monthly assays over 1 year, starting with freshly prepared (time 0 ) plates. Within a given time point, data are plotted in well (row and column) order-A1, A2 . . A48, B1, B2 . . B48 . . AF48. To further reveal data trends, wells are shaped by distance from plate edge - circles for outermost wells and squares for the next ring of wells toward the center.

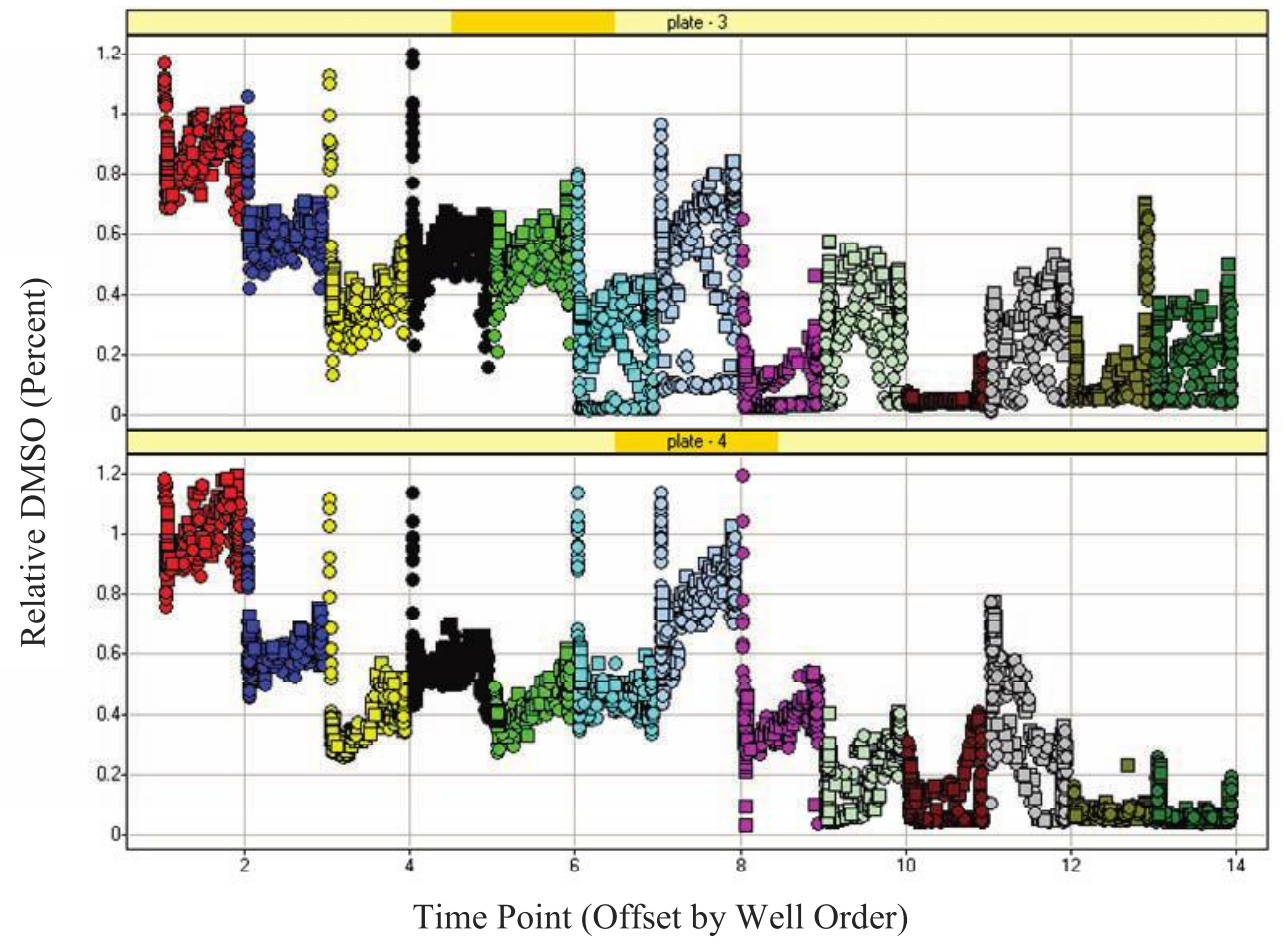

FIG. 4. 1,536-well data from storage at $-20^{\circ} \mathrm{C}$ with a bag. Data are paneled by storage condition, where plate-type 3 data (top) correspond to empty evaporation wells and plate-type 4 data (bottom) to filled evaporation wells. Graph axes, colors, and shapes are as described for Fig. 3. 


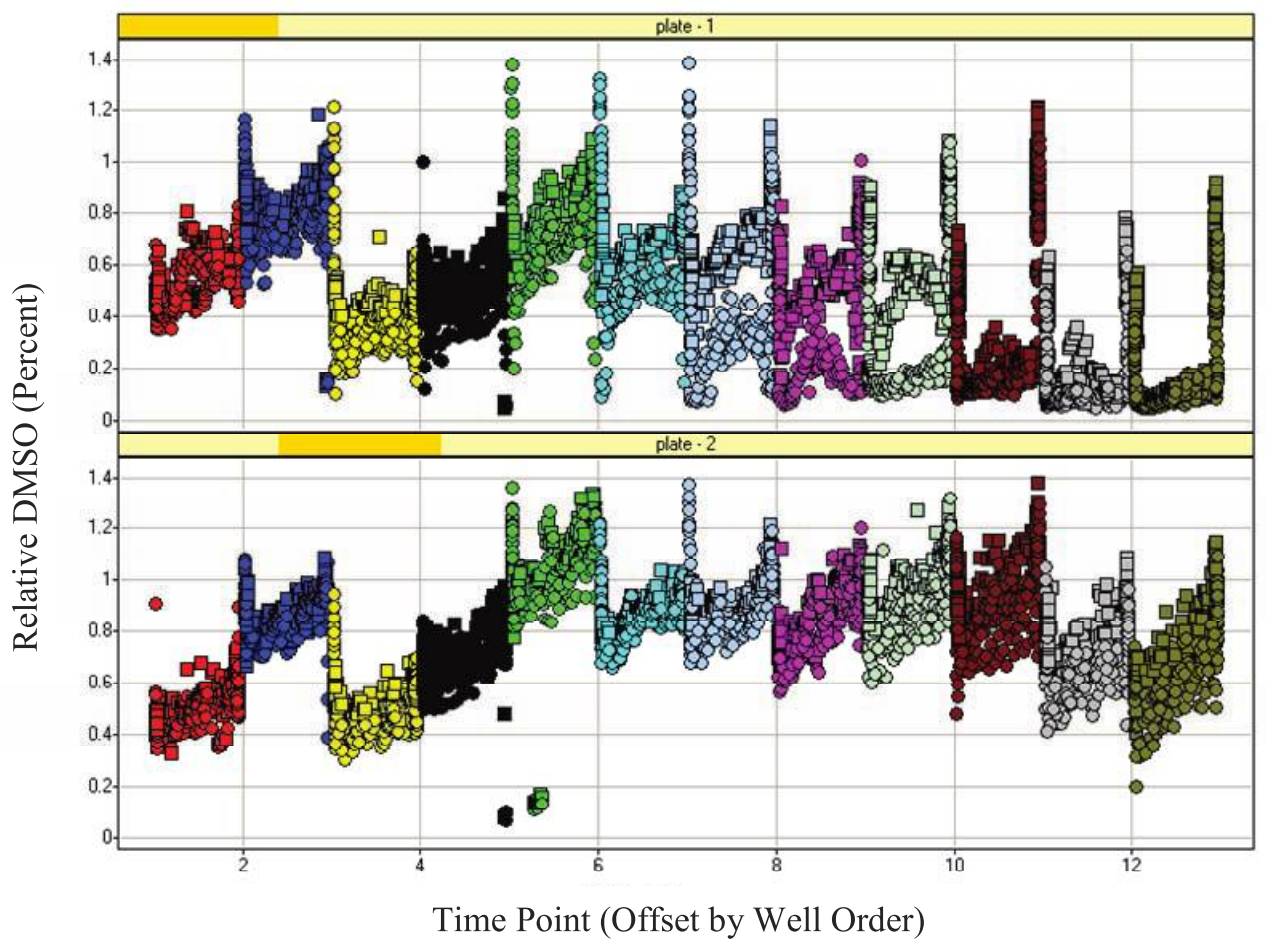

FIG. 5. 3,456-well data from storage at $-20^{\circ} \mathrm{C}$ without a bag. Data are paneled by storage condition, where plate-type 1 data (top) correspond to empty evaporation wells and plate-type 2 data (bottom) to filled evaporation wells. Graph axes, colors, and shapes are as described and depicted for 1,536-well data in Fig. 3, with the exception that the olive-colored pile corresponds to 12 months (not 11) of 3,456-well plate storage; 11-month data were not obtained for 3,456-well studies.

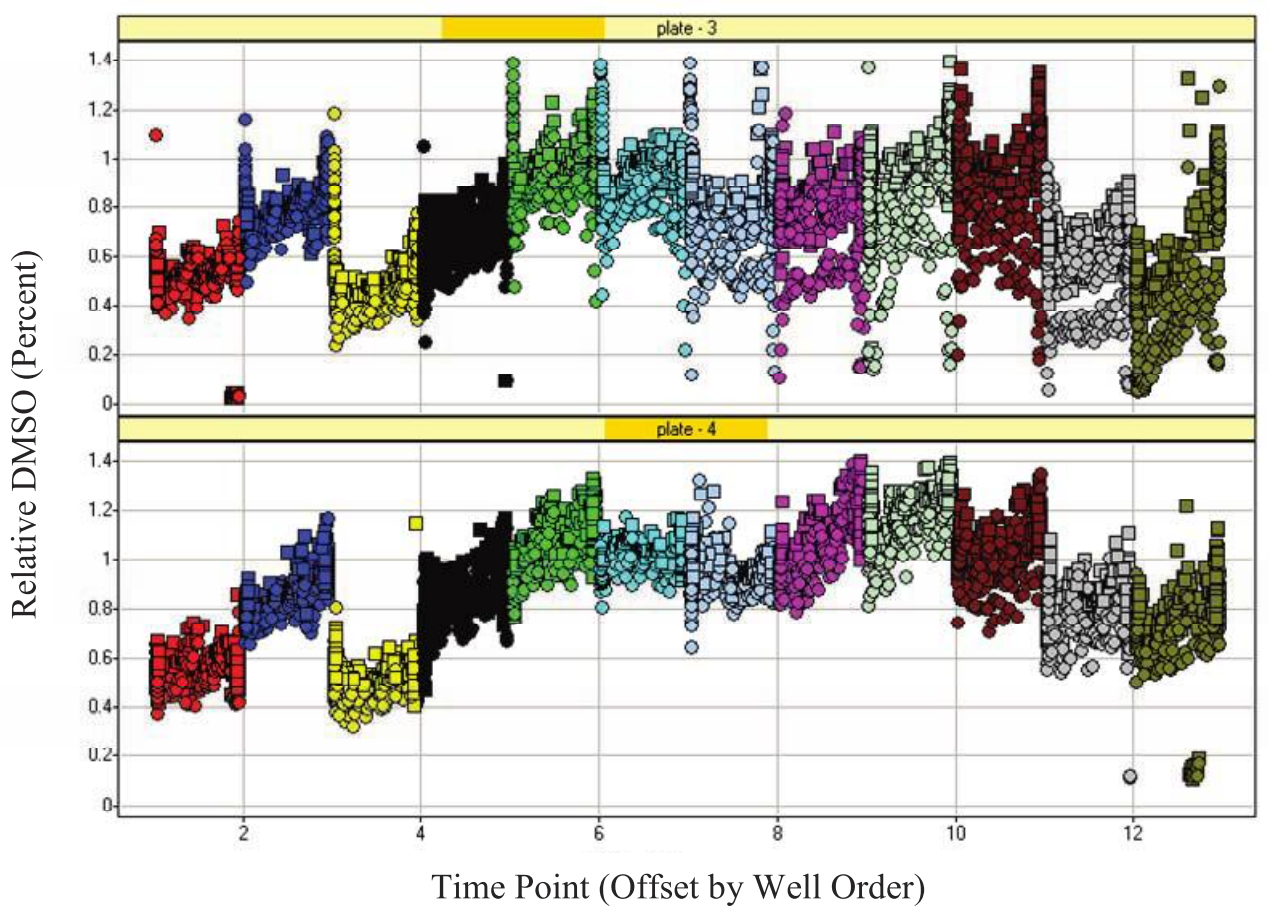

FIG. 6. 3,456-well data from storage at $-20^{\circ} \mathrm{C}$ with a bag. Data are paneled by storage condition, where plate-type 3 data (top) correspond to empty evaporation wells and plate-type 4 data (bottom) to filled evaporation wells. Graph axes, colors, and shapes are as described for Fig. 5. 


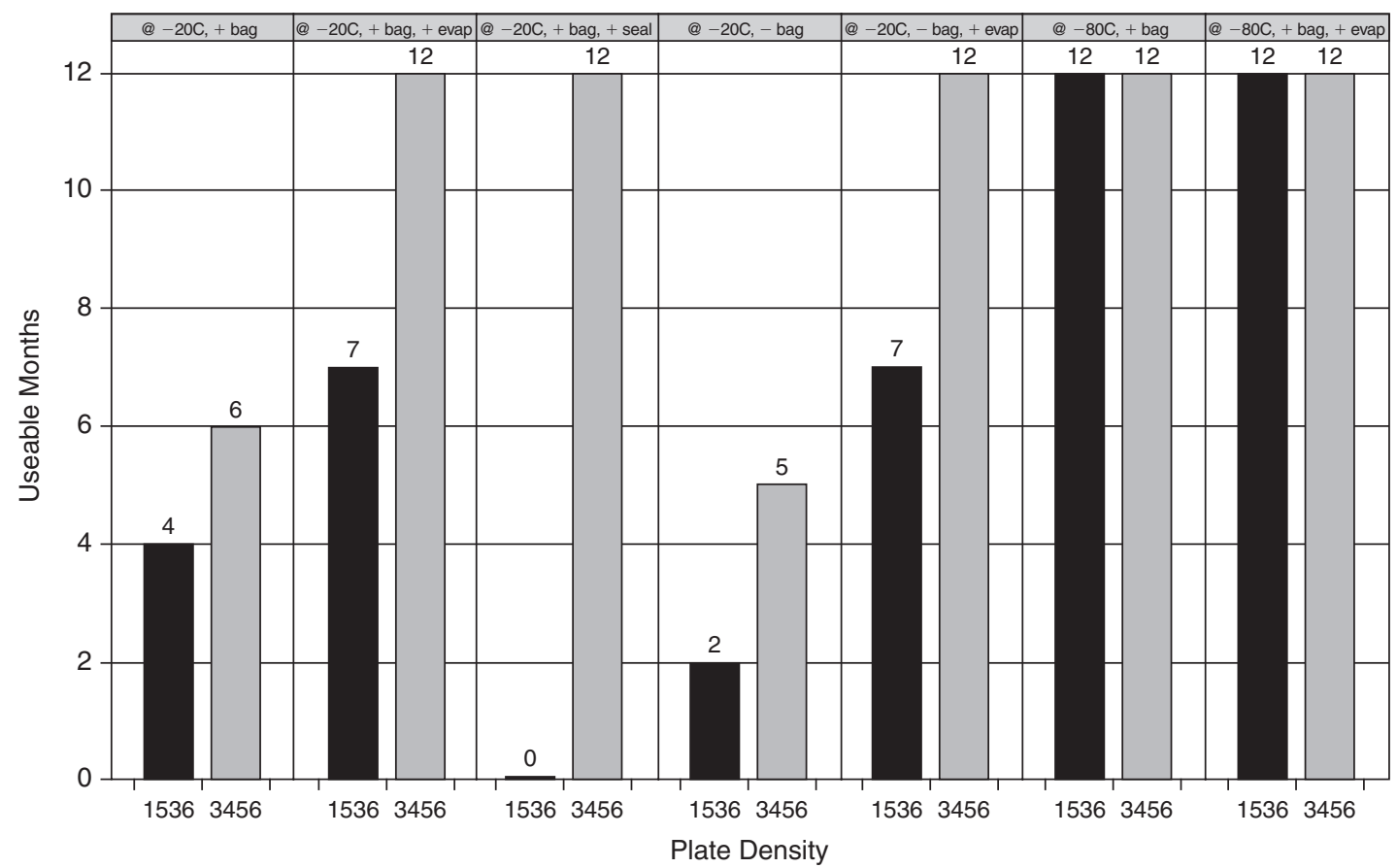

FIG. 7. Summary graph of study results. Data are paneled by storage condition and shaded by plate density. Bars indicate stable storage times (in months) for each condition studied, after which plates would be discarded because of evaporation of a significant portion of plate wells. Note, however, that several conditions were stable for the duration of the study.

worse until month 6 . Beyond that date, variability increased markedly, so use of plates would be discouraged. With filled evaporation wells, bagged plates appeared more consistent across all time points and usable for 12 months, as was found in the 1,536 -well study. The $-20^{\circ} \mathrm{C}$ heat-sealed condition was much more consistent than in the 1,536-well assay, with plate stability apparent for a year as well (data not shown). Storage in a bag at $-80^{\circ} \mathrm{C}$ with or without filled evaporation wells exhibited no discernable evaporation at plate edges over the yearlong study period, as was observed in the 1,536-well study (data not shown). Taken together, the data indicate that for each storage condition evaluated, 3,456-well plates preplated with $10 \mathrm{nl}$ of $75 \% \mathrm{DMSO} / 25 \%$ water can be stored as long as or longer than analogous storage of 20 $\mathrm{nl}$ in 1,536-well plates, thereby highlighting an unexpected advantage to miniaturization. A summary of results appears in Fig. 7.

Berg et al. ${ }^{13}$ have studied physical parameters influencing DMSO behavior in high-density microplates. They noted that high-density microplates have higher surface to volume ratios and are influenced more by hydrophobic repulsion, DMSO hygroscopy, and evaporation of the DMSO-water mix than lower-density plate formats. Moreover, they noted that the distance between the plate surface and liquid surface was a key factor in evaporative effects and that such effects were most significant at higher densities. Table 1 details some calculations based upon ChemLib plate literature. ${ }^{12}$ Since the well heights and surface area to spot volume ratios did not differ appreciably between the two plate types, some other factor must better explain the somewhat enhanced storage times of 3,456-well plates over 1,536-well densities. Indeed, the well volume to spot volume ratio was greatest and surface area to well volume ratio least in

Table 1. Surface Area (SA) and Volume (V) Calculations Based on ChemLib Microplate Literature ${ }^{12}$

\begin{tabular}{lcc}
\hline & \multicolumn{2}{c}{ Well dimension } \\
\cline { 2 - 3 } & 1,536-well plate & 3,456-well plate \\
\hline Well V $(\mu 1)$ & 8.95 & 2.64 \\
Diameter $(\mathrm{mm})$ & 1.70 & 1.13 \\
$\quad$ Top & 1.36 & 0.90 \\
$\quad$ Bottom & 4.85 & 3.25 \\
Depth $(\mathrm{mm})$ & & \\
Circumference $(\mathrm{mm})$ & 5.34 & 3.55 \\
$\quad$ Top & 4.27 & 2.83 \\
$\quad$ Bottom & 23.30 & 10.36 \\
Well area $\left(\mathrm{mm}^{2}\right)$ & 1.45 & 0.64 \\
$\quad$ Side & 24.75 & 10.99 \\
$\quad$ Bottom & 0.02 & 0.01 \\
Total SA $\left(\mathrm{mm}^{2}\right)$ & 447.50 & 264.00 \\
Spot volume $(\mathrm{S})(\mu 1)$ & $1,237.62$ & $1,099.39$ \\
V/S ratio & 2.77 & 4.16 \\
SA/S ratio & & \\
SA/V ratio & & \\
\hline
\end{tabular}

$\mathrm{SA} / \mathrm{S}$ ratios were comparable between the two studies. In contrast, the well $\mathrm{V} / \mathrm{S}$ ratio was greatest and $\mathrm{SA} / \mathrm{V}$ ratio was least in 1,536-well plates. 
1,536-well plates. With less volume for well atmosphere equilibration and more surface area for physical parameters at play, nanospots preplated in 3,456-well plates evaporated less at plate edges than nanospots in 1,536well plates for each comparable storage condition.

\section{Conclusions}

Stored at $-20^{\circ} \mathrm{C}$, without a bag, ChemLib 1,536-well plates (containing $20 \mathrm{nl}$ of $75 \% \mathrm{DMSO} / 25 \%$ water) appeared usable for 2 months. With the addition of evaporation wells, plate-life was extended out to 7 months. Storing plates with a bag but without filled evaporation wells was worse than filling the evaporation wells but not using the bag-plates stored at $-20^{\circ} \mathrm{C}$ with a bag were usable for 4 months and with filled evaporation wells for 7 months. The $-20^{\circ} \mathrm{C}$ heatsealed condition was highly variable, with six good plates and six bad plates in no particular order. Finally, both $-80^{\circ} \mathrm{C}$ conditions exhibited no discernable evaporation at plate edges over the entirety of the 12-month study, likely because of freezing of the $75 \% \mathrm{DMSO} / 25 \%$ water mix.

Stored at $-20^{\circ} \mathrm{C}$, without a bag, ChemLib 3,456-well plates (containing $10 \mathrm{nl}$ of $75 \% \mathrm{DMSO} / 25 \%$ water) were usable for 5 months. With the addition of evaporation wells, it appeared that a full year of storage was possible - even without a bag. Plates stored at $-20^{\circ} \mathrm{C}$ with a bag were usable for up to 6 months or so, beyond which variability increased markedly, but with filled evaporation wells, the outermost assay wells appeared more consistent, and plates usable for all 12 months. The $-20^{\circ} \mathrm{C}$ heat-sealed condition was much more consistent at edges than in the 1,536-well assay, with plate stability apparent for a year as well. Finally, as with the 1,536 -well study, both $-80^{\circ} \mathrm{C}$ conditions exhibited no discernable evaporation from outer assay wells over the entirety of the 12-month study.

Our results highlighted the effectiveness of evaporation well filling for "sacrificial" evaporation and preservation of test compound solvation in the outermost assay-ready plate wells. Moreover, results obtained with unfilled evaporation wells indicated that the ChemLib lid design contributed further to integrity of stored samples - findings that may better apply to those laboratories utilizing fully automated storage, freezer racks, or other inventory systems not amenable to the bagged conditions considered. Finally, because of increased surface area to volume ratios in the 3,456-well format relative to that of the 1,536-well plates, samples could be stored longer in the higher-density format.

\section{Acknowledgments}

The authors wish to thank Jessica Antos for technical assistance and Katrina Schinske for helpful discussions and suggestions.

\section{Disclosure Statement}

No competing financial interests exist.

\section{References}

1. Kozikowski BA, Burt TM, Tirey DA, Williams LE, Kuzmak BR, Stanton DT, et al.: The effect of room-temperature storage on the stability of compounds in DMSO. J Biomol Screen 2003;8:205-209.

2. Kozikowski BA, Burt TM, Tirey DA, Williams LE, Kuzmak BR, Stanton DT, et al.: The effect of freeze/thaw cycles on the stability of compounds in DMSO. $\mathrm{J}$ Biomol Screen 2003;8:210-215.

3. Cheng X, Hochlowski J, Tang H, Hepp D, Beckner C, Kantor S, et al.: Studies on repository compound stability in DMSO under various conditions. J Biomol Screen 2003; 8:292-304.

4. Bowes S, Sun D, Kaffashan A, Zeng C, Chuaqui C, Hronowski X, et al.: Quality assessment and analysis of Biogen Idec compound library. J Biomol Screen 2006; 11:828-835.

5. Ellson R, Stearns R, Mutz M, Brown C, Browning B, Harris D, et al.: In situ DMSO hydration measurements of HTS compound libraries. Comb Chem High Throughput Screen 2005;8:489-498.

6. Oldenburg K, Pooler D, Scudder K, Lipinski C, Kelly M: High throughput sonication: evaluation for compound solubilization. Comb Chem High Throughput Screen 2005;8: 499-512.

7. Harris D, Mutz M, Sonntag M, Kong B, Krueger G, Ellson R: Putting a lid on edge effects: protecting compound libraries with lid-based environmental control. Poster presented at the $12^{\text {th }}$ Annual Conference of the Society for Biomolecular Screening, Seattle, WA, September 2006.

8. Kornienko O, Lacson R, Kunapuli P, Schneeweis J, Hoffman I, Smith T, et al.: Miniaturization of whole live cellbased GPCR assays using microdispensing and detection systems. J Biomol Screen 2004;9:186-195.

9. Yasgar A, Shinn P, Jadhav A, Auld D, Michael S, Zheng $\mathrm{W}$, et al.: Compound management for quantitative highthroughput screening. JALA Charlottesv Va 2008; 13:79-89.

10. Rassmussen DH, MacKenzie AP: Phase diagram for the system water-dimethylsulphoxide. Nature 1968;220: 1315-1317.

11. Invitrogen Corp.: Vivid ${ }^{\circledR}$ CYP3A4 Screening Kit Protocol. Invitrogen Corp., Carlsbad, CA, 2005.

12. Aurora Discovery, Inc.: ChemLib ${ }^{\mathrm{TM}} 1536$-Well and 3456Well Microplates. Aurora Discovery, San Diego, CA, 2005.

13. Berg M, Undisz K, Thiericke R, Zimmerman P, Moore T, Posten C: Evaluation of liquid handling conditions in microplates. J Biomol Screen 2001;6:47-56.

Address reprint requests to: Scott C. Wise

Deciphera Pharmaceuticals 4950 Research Park Way Lawrence, KS 66047

E-mail: swise@deciphera.com 